\title{
INDICADORES DA SÍNDROME DE BURNOUT EM ATLETAS AMADORES BRASILEIROS DE RUGBY SEVENS
}

\author{
Elisângela Maranin da Silva \\ Metrocamp, , Campinas, São Paulo, Brasil \\ João Guilherme Cren Chiminazzo \\ Universidade Estadual de Campinas, Campinas, São Paulo, Brasil \\ Daniel Alvarez Pires \\ Universidade Federal do Pará, Castanhal, Pará, Brasil
}

\begin{abstract}
Resumo
Os objetivos do estudo foram mensurar como a síndrome de burnout afeta atletas brasileiros de rugby sevens e verificar o efeito da variável sexo na percepção da síndrome. Participaram do estudo 21 atletas amadores (11 homens e dez mulheres), que responderam a uma ficha de dados demográficos e ao Questionário de Burnout para Atletas. Para análise de dados utilizou-se a estatística descritiva e a estatística inferencial (teste U de Mann-Whitney). Os atletas avaliados apresentaram índices baixos e moderados nas dimensões de burnout e no burnout total. Atletas do sexo feminino apresentaram maior percepção da dimensão "reduzido senso de realização esportiva" do que seus pares do sexo masculino. Não foi verificado o efeito da variável sexo para as demais dimensões e para o burnout total.
\end{abstract}

Palavras-chave: Psicologia; Esgotamento Profissional; Futebol Americano.

\section{Introdução}

A síndrome de burnout é um objeto de estudo recente na Psicologia em geral e na Psicologia do Esporte em particular. O primeiro autor a mencioná-la no âmbito da Psicologia geral foi Freudenberger (1975), que a definiu como a exaustão advinda do excesso de demandas de energia, força ou recursos. Para Maslach (2005), burnout é uma síndrome caracterizada pelo esgotamento físico, psíquico e emocional, em decorrência de trabalho estressante e excessivo, feito de maneira crônica, podendo levar o indivíduo a uma desistência do seu trabalho.

De acordo com Trigo, Teng e Hallak (2007), o burnout foi reconhecido como um risco ocupacional para profissões que envolvem cuidados com saúde, educação e serviços humanos. A síndrome de burnout está catalogada com o código Z73.0 no grupo V da Classificação Internacional das Doenças - CID-10.

Segundo Maslach (2005), o burnout deve ser entendido como uma síndrome, ou seja, um conjunto de sintomas e está fundamentado em três dimensões: a) exaustão emocional, que é caracterizada por sentimentos de extrema fadiga; b) despersonalização, representada por atitudes e sentimentos negativos com respeito aos clientes, sendo ilustrada por um comportamento impessoal, de desligamento e descuido em relação aos mesmos; c) e a reduzida satisfação profissional, que reflete avaliações negativas sobre si mesmo, particularmente com referência à habilidade de obter sucesso no trabalho com clientes. 
Após a síndrome de burnout ser identificada em profissões de ajuda, a mesma passou a ser observada no meio do esporte e da Educação Física. Profissionais como os professores de Educação Física, treinadores, preparadores físicos, atletas e árbitro se constituíam em indivíduos propensos à aquisição dessa síndrome. Para Smith (1986), o burnout no esporte é uma reação ao estresse crônico, e possui componentes físicos, mentais e comportamentais, apresentando como característica mais marcante a saturação psicológica, emocional e, por vezes, física de uma atividade anteriormente agradável e procurada. Chiminazzo e Montagner (2009) abordaram as implicações da síndrome no treinamento esportivo e apontam que a queda de rendimento pode ser um sintoma de burnout. No ambiente esportivo, a exposição ao estresse crônico por um longo período de tempo torna o indivíduo mais suscetível aos sintomas da síndrome de burnout que podem levar ao abandono da prática da modalidade esportiva (COSTA et al., 2012; PIRES et al., 2012).

Essas investigações sobre a síndrome de burnout no esporte se justificam por três motivos: (1) o impacto da síndrome no desempenho atlético; (2) a necessidade de manutenção da saúde e qualidade de vida dos atletas; e (3) a necessidade de entender melhor o fenômeno, suas consequências e formas de prevenção (PIRES et al., 2012).

Em relação à modalidade rugby sevens, estudo recente inferiu que a prescrição adequada dos treinamentos é determinante para evitar o surgimento do burnout (SOBRAL et $a l ., 2014)$. Outro fator relevante para a prevenção do burnout em atletas de rugby é a adoção de estratégias de controle do perfeccionismo, pois esse tipo de comportamento se constitui em um preditor da síndrome (HILL; APPLETON, 2011). Os indicadores de burnout em atletas dessa modalidade foram investigados em âmbito internacional (CRESSWELL; EKLUND, 2006) e nacional (SOBRAL et al., 2014), com o registro de percepções reduzidas da síndrome e de suas dimensões. Entretanto, tais estudos tiveram como participantes somente atletas do sexo masculino. Consequentemente, não foram reportados dados referentes aos indicadores da síndrome em atletas do sexo feminino. A discussão a respeito das possíveis diferenças nas percepções de burnout entre atletas homens e mulheres apresenta uma lacuna na literatura nacional e internacional. Os poucos registros encontrados se referem a uma modalidade individual e uma coletiva, casos do tênis (PIRES et al., 2013) e do basquetebol (PIRES; SOUZA; CRUZ, 2010), cujas investigações em atletas brasileiros não identificaram diferenças significativas entre os sexos.

Diante do exposto, o presente estudo teve como objetivo mensurar a síndrome de burnout em atletas brasileiros de rugby e verificar o efeito da variável sexo na incidência da síndrome entre atletas.

\section{Material e Métodos}

\section{Amostra e Cuidados Éticos}

Participaram do estudo 21 atletas amadores de rugby sevens de ambos os sexos, pertencentes à categoria adulta, sendo 11 homens e dez mulheres, com faixa etária de 12 a 34 anos de idade, com média de 22,24 \pm 5,85 anos. Os atletas menores de idade participam das competições na categoria adulta, com as devidas autorizações de seus pais e técnicos do clube, sem que isso acarrete algum dano ao seu estado físico ou emocional. Possuem tempo de prática de oito meses a sete anos, com média de 19,5 $\pm 49,09$ meses, e frequência média de $2,70 \pm 1,29$ treinos por semana, sendo $2,55 \pm 1,33$ para o feminino e 2,90 $\pm 1,30$ para o masculino. Os participantes não se dedicam apenas à carreira atlética, possuindo atividades paralelas como trabalho e estudo. 
Todos os participantes foram informados sobre os procedimentos que seriam realizados nas coletas de dados e, previamente à sua participação, assinaram o Termo de Consentimento Livre e Esclarecido, conforme a resolução no 466/2012 do Conselho Nacional de Saúde do Brasil para experimentos com humanos, concordando em participar dos procedimentos metodológicos propostos na pesquisa.

\section{Instrumentos}

Para caracterização geral dos atletas foi utilizada uma ficha de dados sociodemográficos contendo dados referentes ao sexo, idade, tempo de prática, categoria, período (pré-temporada, temporada, pós-temporada), tempo de duração e frequência semanal de treinamentos. Para análise da síndrome de burnout, foi utilizado o Athlete Burnout Questionnaire (ABQ), de autoria de Raedeke e Smith (2001), cuja validação para o idioma português foi realizada por Pires, Brandão e Silva (2006), originando o Questionário de Burnout para Atletas (QBA). O QBA tem como objetivo mensurar as três dimensões de burnout: a) exaustão física e emocional; b) a desvalorização esportiva; e c) o reduzido senso de realização esportiva.

O questionário é composto por 15 itens, sendo cinco itens correspondentes para cada dimensão. As respostas são representadas em uma escala tipo Likert que varia de "Quase Nunca" (1) a "Quase Sempre" (5), tendo como frequência intermediária as seguintes: "Raramente" (2), "Algumas Vezes" (3) e "Frequentemente" (4). Os resultados são obtidos a partir da média aritmética das respostas dadas aos cinco itens correspondentes a cada dimensão de burnout. O valor de burnout total é calculado pela média dos 15 itens do QBA. A interpretação dos escores se dá através da utilização da variação de frequência de sentimentos, isso quer dizer que, se um atleta obtém uma média de 3,5 para a dimensão desvalorização esportiva, considera-se que esse atleta possui sentimento relacionado a tal dimensão com frequência de algumas vezes a frequentemente. A Tabela 1 apresenta as frequências e seus intervalos referentes à percepção das dimensões de burnout e de burnout total (RAEDEKE; SMITH, 2001).

Tabela 1 - Frequências ou intervalos de frequência referentes às percepções das dimensões de burnout e do burnout total.

\begin{tabular}{cc}
\hline Valor obtido no QBA & Frequência ou intervalo de frequência \\
\hline 1 & Quase nunca \\
1,01 a 1,99 & Quase nunca a raramente \\
2 & Raramente \\
2,01 a 2,99 & Raramente a algumas vezes \\
3 & Algumas vezes \\
3,01 a 3,99 & Algumas vezes a frequentemente \\
4 & Frequentemente \\
4,01 a 4,99 & Frequentemente a quase sempre \\
5 & Quase Sempre \\
\hline
\end{tabular}




\section{Procedimentos}

Por questão de logística, a realização da coleta de dados foi feita numa competição de rugby sevens com sede na cidade de Indaiatuba-SP. Essa competição contou com a participação de atletas de diferentes estados do Brasil. A coleta de dados foi realizada no segundo dia de competição, quando os atletas foram abordados no intervalo entre as partidas e foram informados a respeito dos procedimentos para se responder a ficha sociodemográfica e o QBA. O tempo para o preenchimento da ficha e dos itens do instrumento foi de aproximadamente 15 minutos.

\section{Análise de Dados}

Para a análise de dados da síndrome de burnout, foi utilizada a estatística descritiva a partir da obtenção da média e desvio padrão. Os escores encontrados foram interpretados através da utilização dos intervalos de frequência de sentimentos (ver Tabela 1).

Em seguida foi utilizado o teste de Shapiro-Wilk para verificar a normalidade dos dados. Para a comparação dos escores entre os diferentes sexos foi utilizado um teste de comparação de postos (teste U de Mann Whitney). Os dados foram tratados pelo software SPSS 19.0. O índice de significância adotado foi $\mathrm{p}<0,05$.

\section{Resultados}

O gráfico 1 apresenta os escores médios das dimensões de burnout e do burnout total dos atletas de rugby.

Gráfico 1- Escores das dimensões de burnout e do burnout total em ambos os sexos $(\mathrm{n}=21)$.

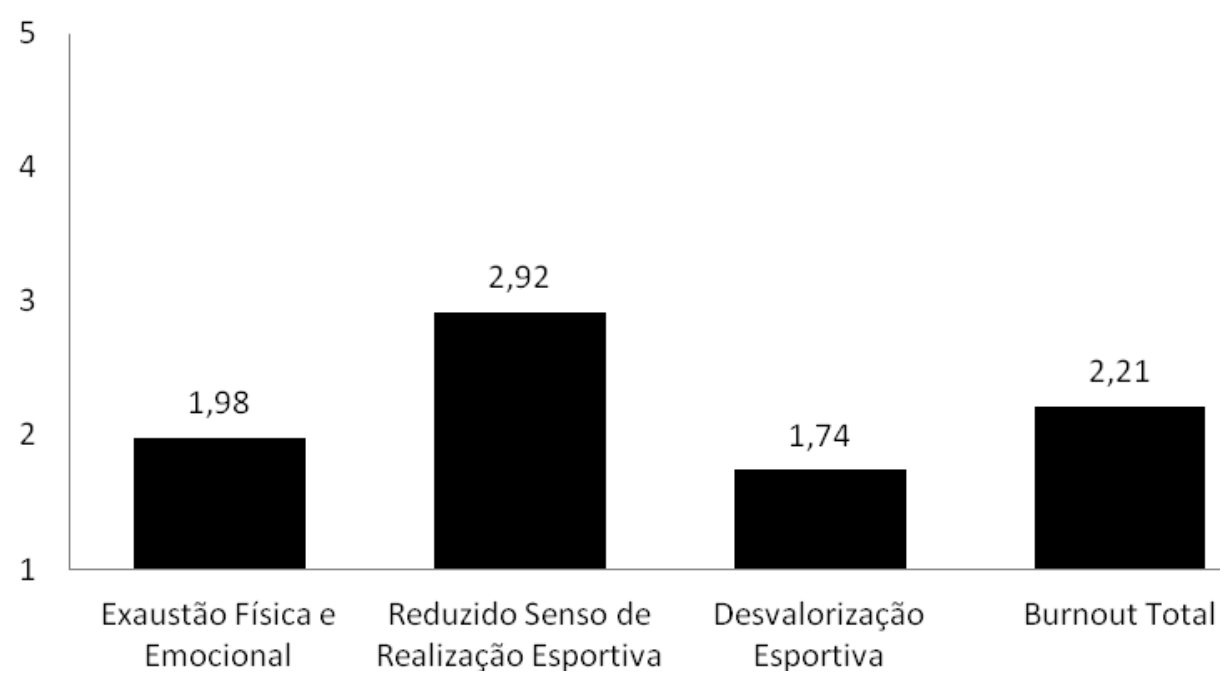

Observa-se que as dimensões exaustão física e emocional e desvalorização esportiva apresentaram escores médios correspondentes a um intervalo de frequência entre quase nunca a raramente. Para as dimensões "reduzido senso de realização esportiva e burnout total", os escores médios encontram-se com intervalos de frequência entre raramente a algumas vezes.

O teste de normalidade apontou para uma distribuição não paramétrica das variáveis de burnout, o que determinou o emprego do teste U de Mann-Whitney para a verificação de 
significância na comparação entre os sexos. Por se tratar de um teste não paramétrico, não foram utilizadas as médias dos escores, mas sim as medianas. O Gráfico 2 apresenta as medianas de burnout total e suas dimensões entre atletas homens e mulheres.

Gráfico 2 - Medianas de burnout total e suas dimensões em atletas de rugby dos sexos masculino $(\mathrm{n}=11)$ e feminino $(\mathrm{n}=10) . * \mathrm{p}<0,05$

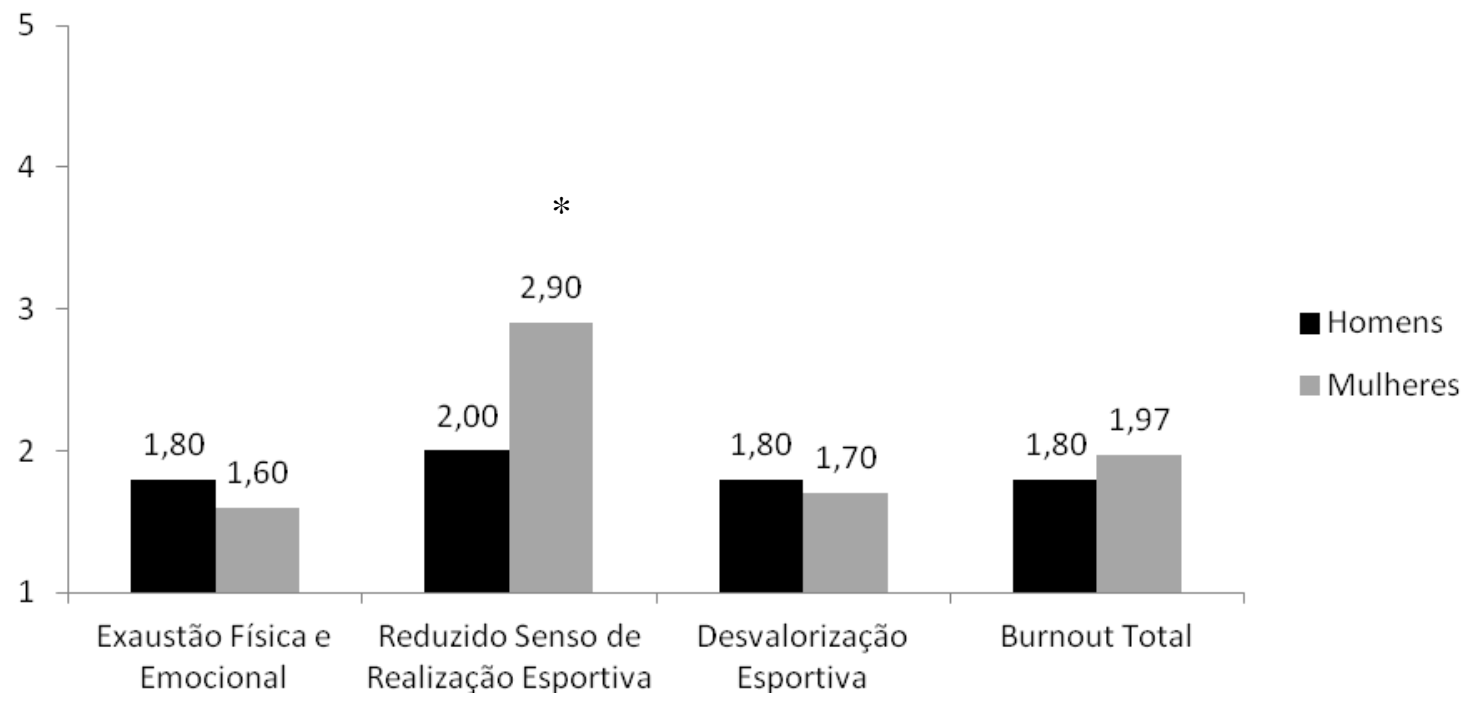

Em relação à dimensão exaustão física e emocional, tanto os atletas do sexo masculino quanto seus pares do sexo feminino obtiveram medianas correspondentes a um intervalo de frequência entre quase nunca a raramente. Não foi encontrada diferença significativa $(p=0,73)$ por sexo na percepção dos atletas em relação a essa dimensão.

Quanto às percepções de reduzido senso de realização esportiva, a mediana encontrada para o sexo masculino corresponde à frequência raramente, enquanto que o grupo feminino apresentou mediana correspondente a um intervalo de frequência entre raramente e algumas vezes. Foi encontrada diferença significativa $(\mathrm{p}=0,01)$ por sexo na percepção dos atletas em relação a essa dimensão.

Os achados relacionados à dimensão "desvalorização esportiva" apontaram medianas para homens e mulheres referente a um intervalo de frequência entre quase nunca a raramente. Não foram encontradas diferenças significativas $(p=0,44)$ por sexo na percepção dos atletas em relação a essa dimensão.

A mediana de burnout total do grupo feminino foi maior do que a do grupo masculino, porém ambas estão relacionadas ao intervalo de frequência compreendido entre raramente e algumas vezes. Apesar dos resultados terem apontado que o grupo feminino apresentou uma pequena superioridade em relação ao grupo masculino, não foram encontradas diferenças significativas $(\mathrm{p}=0,25)$ ao se confrontar os valores de burnout total obtidos.

\section{Discussão}

O presente estudo teve como objetivo mensurar como a síndrome de burnout afeta atletas brasileiros de rugby e verificar o efeito da variável sexo na incidência da síndrome entre atletas.

Ao se analisar a dimensão "exaustão física e emocional", foi possível observar um baixo escore médio dos atletas de ambos os sexos. Para Raedeke (1997), essa dimensão está 
associada a altas demandas de treinos e competições. Assim, consideramos que os resultados obtidos se justificam pelo fato de os atletas avaliados não possuírem uma demanda intensa de treinos e competições, pois desenvolvem outras atividades paralelas, diferenciando-se de atletas profissionais, os quais se dedicam exclusivamente ao esporte. Essa dimensão também tem sido associada à presença da lesão em atletas de rugby (CRESSWELL; EKLUND, 2006), pois o processo de recuperação das lesões mais frequentes na modalidade costuma ser lento. Como os participantes do presente estudo estavam participando de competição, sem apresentar ocorrência de lesão, tais fatos corroboram os indicadores de reduzida percepção de exaustão física e emocional.

A dimensão "reduzido senso de realização esportiva" obteve maior pontuação entre todas as dimensões, o que pode ser um indicativo de que os atletas não estão plenamente satisfeitos em relação às suas habilidades, destrezas e realizações no esporte (RAEDEKE; SMITH, 2001), fato comum em atletas amadores, que ainda estão distantes de atingirem o ápice de sua carreira profissional (GOULD et al., 1997). Os resultados podem ser explicados pelo fato de que muitos atletas que se encontram na categoria amadora não têm a oportunidade de alcançar o nível profissional, tendo em vista as dificuldades que enfrentam devido ao pouco investimento do país no esporte, principalmente em modalidades ainda incipientes como o rugby sevens. Além disso, fatores como a queda de rendimento em virtude das cobranças de pais e treinadores podem influenciar o desempenho do atleta, contribuindo para o sentimento de insatisfação e ausência de êxito na modalidade. Sintomas dessa dimensão são a falta de progressos no desempenho atlético e percepções de falta de sucesso e falta de talento (GOULD et al., 1996). No período competitivo, parte da temporada na qual os dados do presente estudo foram coletados, atletas de rugby apresentaram elevação na percepção de reduzido senso de realização esportiva em comparação com o período précompetitivo (CRESSWELL; EKLUND, 2006).

A dimensão "desvalorização esportiva" se refere à despreocupação, à falta de desejo e de interesse em relação ao esporte. Nesse cenário, o atleta passa a se comportar de modo impessoal e insensível, demonstrando pouco ou nenhuma preocupação com seu rendimento e com o seu envolvimento no esporte (RAEDEKE; SMITH, 2001). Entre as dimensões apresentadas, a desvalorização esportiva apresentou menor escore médio entre os atletas, o que pode ser um indicativo de que o grupo investigado demonstra compromisso e envolvimento em relação à modalidade esportiva. Os resultados obtidos evidenciam a motivação e satisfação que os atletas possuem em relação à prática do rugby, elementos importantes para que o surgimento do burnout seja evitado (CRESSWELL; EKLUND, 2005).

Os atletas apresentaram valores moderadamente reduzidos tanto para o burnout total quanto para as suas dimensões, fatores indicativos de que os mesmos não apresentavam manifestações significativas da síndrome. Indicadores reduzidos de burnout também foram observados em jogadores amadores de rugby sevens no Estado da Paraíba (SOBRAL et al., 2014) e pertencentes à união amadora de rugby da Nova Zelândia (CRESSWELL; EKLUND, 2005). Ainda no âmbito das modalidades coletivas, foram observados valores moderados a reduzidos das dimensões de burnout em atletas brasileiros de basquetebol amador (PIRES: SOUZA; CRUZ, 2010), do circuito nacional de vôlei de praia (VIEIRA et al., 2013) e de futebol das categorias profissional e júnior (VERARDI et al., 2015). Tal constatação está de acordo com os pressupostos de Smith (1986), que afirma que os sentimentos inerentes à síndrome são geralmente menos notados em atletas de esportes coletivos. Isso se deve, principalmente, à divisão de funções e tarefas, além do maior número de relações interpessoais existentes nos esportes de equipe.

Estudos epidemiológicos têm apontado uma incidência variável de burnout entre 1 a $10 \%$ dos atletas investigados (GUSTAFSSON et al., 2007; MARTÍNEZ; GÓMEZMÁRMOL, 2014). Apesar dos reduzidos valores observados, o atleta, em muitos casos, não 
percebe que está esgotado física e emocionalmente. Porém, evidenciam-se sintomas que o incomodam ao longo da prática esportiva. Nesse cenário, torna-se imprescindível o papel dos integrantes da comissão técnica (em especial o psicólogo ou psicóloga do esporte) no reconhecimento e tratamento desse estado psicofisiológico prejudicial, prevenindo o abandono esportivo (CHIMINAZZO; MONTAGNER, 2009).

Em relação à verificação do efeito da variável sexo sobre a percepção da síndrome de burnout, cada dimensão será discutida individualmente, pois apesar de serem componentes do mesmo construto psicológico, possuem características particulares e se diferenciam entre si.

Ao se analisar a dimensão exaustão física e emocional, a ausência de diferença significativa entre os sexos diverge da perspectiva de que as mulheres possuem percepções mais frequentes dessa dimensão por serem mais emotivas, mais envolvidas com o cuidado, alimentação e preocupação com o bem estar do próximo (SILVA; CARLOTO, 2003). Não foram encontradas evidências na literatura a respeito de comparações das dimensões de burnout entre os sexos na população de atletas. Na perspectiva dos treinadores, as mulheres apresentam maiores escores do que os homens nessa dimensão (GOODGER et al., 2007).

Quanto às percepções de reduzido senso de realização esportiva, foi percebida superioridade no escore do grupo feminino. Esse achado pode estar associado à maior percepção de competência percebida pelos adolescentes do sexo masculino evidenciada por Jacobs et al. (2002). Como uma parcela dos participantes do presente estudo é composta por adolescentes ou adultos jovens, é possível que tal percepção de competência ainda esteja acentuada entre os homens, sugerindo a maior percepção de reduzido senso de realização esportiva entre as atletas do sexo feminino. Há a hipótese ainda de que os achados justificamse devido ao grupo feminino ser composto por atletas amadoras que, no momento da avaliação, estavam sem técnico e não possuíam resultados expressivos na modalidade.

Diferentemente dos resultados obtidos para a dimensão "reduzido senso de realização esportiva", não foi verificado o efeito da variável sexo sobre a percepção da dimensão "desvalorização esportiva". Em oposição aos achados do presente estudo, Isoard-Gautheur et al. (2015) verificaram maior percepção dessa dimensão em jovens atletas de handebol do sexo feminino. De acordo com os autores, a percepção mais elevada de desvalorização esportiva pode representar para as mulheres tanto uma perspectiva desfavorável quanto outra favorável. Por um lado, significa maior dificuldade para a manutenção do engajamento na prática esportiva competitiva. Por outro lado, reflete a avaliação cognitiva da atleta na direção de explorar e incorporar novas identidades sociais, como a convivência no trabalho, com os amigos e com a família de modo a construir um desenvolvimento multidimensional (COAKLEY, 1992).

Não foram encontradas diferenças significativas por sexo relacionadas ao burnout total. Esse achado converge com a meta-análise de Goodger et al. (2007), que avaliaram 27 estudos sobre burnout em atletas e não consideraram a variável sexo como interveniente na percepção da síndrome. Para além do contexto esportivo, estudo no ambiente educacional também não apresentou diferenças entre professores homens e mulheres (SILVA; CARLOTO, 2003).

Ressalta-se, como limitação, o processo de corte transversal desta avaliação em que os atletas foram submetidos, sendo que para uma análise mais minuciosa torna-se importante avaliar o burnout e suas dimensões numa perspectiva longitudinal que contemple também outras técnicas de diagnóstico tais como entrevistas qualitativas, autoconfrontação de imagens e análise do meio ambiente em que estão inseridos. Costa et al. (2012) reforçam que a avaliação do burnout através de diferentes técnicas permite um diagnóstico mais preciso e exato sobre a síndrome. 
Outra limitação consiste no fato de o burnout estar localizado no extremo de um contínuo que se inicia com reações negativas de adaptações ao estresse denominadas de esgotamento e passa pelo overtraining (SILVA, 1990), existe uma limitação em se encontrar atletas em atividade com escores acentuados da síndrome, pois há uma grande possibilidade dos mesmos já terem abandonado o esporte, visto que o abandono é uma das principais consequências do burnout (GUSTAFSSON et al., 2007).

\title{
Conclusão
}

Conclui-se que os atletas avaliados, tanto no grupo masculino quanto no grupo feminino, possuem índices reduzidos de burnout total e suas dimensões "exaustão física e emocional, reduzido senso de realização esportiva e desvalorização esportiva". Os resultados estão de acordo com a literatura, para modalidades coletivas, em virtude da divisão de funções e do maior número de relações interpessoais.

Foi observado o efeito da variável sexo somente na percepção da dimensão reduzido senso de realização esportiva, na qual as atletas mulheres apresentaram percepção mais elevada. Esse indicativo pode estar associado à maior percepção de competência e de talento esportivo entre os atletas homens.

\section{BURNOUT SYNDROME INDICATORS IN BRAZILIAN AMATEUR ATHLETES OF RUGBY SEVENS}

\begin{abstract}
The aims of the study were to measure how the burnout syndrome affects Brazilian rugby sevens athletes and verify the effect of the sex variable in the perception of the syndrome. 21 amateur athletes participated (11 men and 10 women), who responded to a demographic profile and the Burnout Questionnaire for Athletes. For data analysis we used descriptive statistics and inferential statistics (Mann-Whitney U test). The athletes evaluated presented low and moderate levels in the dimensions of burnout and total burnout. Female athletes had higher perception of "reduced sense of sport accomplishment" scale than their male counterparts. The effect of the gender variable to the other dimensions and total burnout was not verified.
\end{abstract}

Keywords: Psychology; Burnout; Professional; Rugby.

\section{INDICADORES DEL SÍNDROME DE BURNOUT EN DEPORTISTAS AFICIONADOS BRASILEÑOS DE RUGBY SEVENS}

\section{Resumen}

Los objetivos del estudio fueron mensurar como el síndrome de burnout afecta deportistas brasileños de rugby sevens y verificar el efecto de la variable de género en la percepción del síndrome. Los participantes fueron 21 atletas aficionados (11 hombres y 10 mujeres), que respondieron a una ficha de datos demográficos y al Cuestionario de Burnout para Deportistas. Para el análisis de datos se utilizó la estadística descriptiva y la estadística inferencial (prueba U de Mann-Whitney). Los deportistas evaluados presentaron niveles bajos y moderados en las dimensiones de burnout y en el burnout total. Las mujeres deportistas 
presentaron mayor percepción del "reducido sentido de logro deportivo" que sus homólogos masculinos. El efecto de la variable de género a las otras dimensiones y para el burnout total no se verificó.

Palabras clave: Psicología; Agotamiento Profesional; Fútbol Americano.

\section{Referências}

CHIMINAZZO, J.; MONTAGNER, P. Síndrome de burnout e esporte: a visão dos técnicos de tênis de campo. Revista da Educação Física, Maringá, v. 20, n. 2, p. 217-223, 2009.

COAKLEY, J. J. Burnout among adolescent athletes: a personal failure or social problem? Sociology of Sport Journal, Champaign, v. 9, n. 3, p. 271-285, 1992.

COSTA, V. et al. Comparação dos níveis de estresse, recuperação e burnout em treinadores de futsal e futebol brasileiros através do RESTQ-COACH. Motricidade, Santa Maria de Feira, v. 8, n. S2, p. 937-945, 2012.

CRESSWELL, S. L.; EKLUND, R. C. Changes in Athlete Burnout Over a Thirty-Week "Rugby Year". Journal of Science and Medicine in Sport, Amsterdã, v. 9, p. 125-134, 2006.

CRESSWELL, S. L.; EKLUND, R. C. Motivation and Burnout among Top Amateur Rugby Players. Medicine and Science in Sports and Exercise, Indianápolis, v. 37, n. 3, p. 469-477, 2005.

FREUDENBERGER, $\mathrm{H}$. The staff burn-out syndrome in alternative institutions. Psychotherapy: theory, research and practice, Hoboken, v. 12, n. 1, p. 73-82, 1975.

GOODGER, K. et al. Burnout in Sport: a systematic review. The Sport Psychologist, Champaign, v. 21, p. 127-151, 2007.

GOULD, D. et al. Burnout in Competitive Junior Tennis Players: I. A Quantitative Psychological Assessment. The Sport Psychologist, Champaign, v. 10, n. 4, p. 322-340, 1996.

GOULD, D. et al. Burnout in Competitive Junior Tennis Players: III. Individual Differences in the Burnout Experience. The Sport Psychologist, Champaign, v. 11, n. 3, p. 257-276, 1997.

GUSTAFSSON, H. et al. Prevalence of Burnout in Competitive Adolescent Athletes. The Sport Psychologist, Champaign, v. 21, n. 1, p. 21-27, 2007.

HILL, A.; APPLETON, P. The predictive ability of the frequency of perfectionistic cognitions, self-oriented perfectionism, and socially prescribed perfectionism in relation to symptoms of burnout in youth rugby players. Journal of Sport Sciences, Abingdon, v. 29, n. 7, p. 695-703, 2011. 
ISOARD-GAUTHEUR, S. et al. Development of Burnout Perceptions during Adolescence among High Level Athletes: a developmental and gendered perspective. Journal of Sport and Exercise Psychology, Champaign, v. 37, p. 436-448, 2015.

JACOBS, J. et al. Changes in 15 children's self-competence and values: gender and domain differences across grades one through twelve. Child Development, Malden, v. 73, n. 2, p. 509-527, 2002.

MARTÍNEZ, B.; GÓMEZ-MÁRMOL, A. Prevalencia del síndrome de burnout en 18 tenistas según su orientación motivacional. Revista Iberoamericana de Psicología del Ejercicio y el Deporte, Las Palmas de Gran Canaria, v. 9, n. 1, p. 111-122, 2014.

MASLACH, C. Entendendo o burnout. In: ROSSI, A.; SAUTER, S. (Org.) Stress e Qualidade de Vida no Trabalho. São Paulo: Atlas, 2005, p. 41-55.

PIRES, D.; BRANDÃO, M.; SILVA, C. Validação do Questionário de Burnout para Atletas. Revista da Educação Física, Maringá, v. 17, n. 1, p. 27-36, 2006.

PIRES, D. et al. A síndrome de burnout no esporte brasileiro. Revista da Educação Física, Maringá, v. 23, n. 1, p. 131-139, 2012.

PIRES, D. et al. Incidência de burnout em tenistas infatojuvenis. In: Congresso Brasileiro de Ciências do Esporte (CONBRACE), 18, 2013, Brasília. Anais ... Brasília, 2013.

PIRES, D.; SOUZA, I.; CRUZ, H. Indicadores da síndrome de burnout e suas subescalas em atletas de basquetebol dos sexos masculino e feminino da seleção municipal de Castanhal-PA. Corpoconsciência, Santo André, v. 14, n. 1, p. 35-48, 2010.

RAEDEKE, T. Is athlete burnout more than just stress? A sport commitment perspective. Journal of Sport and Exercise Psychology, Champaign, v. 19, n. 4, p. 396-417, 1997.

RAEDEKE, T.; SMITH, A. Development and preliminary validation of an athlete burnout measure. Journal of Sport and Exercise Psychology, Champaign, v. 23, n. 4, p. 281-306, 2001.

SILVA, G. N.; CARLOTO, M. S. Síndrome de Burnout: um estudo com professores da rede pública. Psicologia Escolar e Educacional, Maringá, v. 7, n. 2, 145-153, 2008.

SILVA, J. M. An analysis of training stress syndrome in competitive athletics. Journal of Applied Sport Psychology, London, v. 2, p. 5-20, 1990.

SMITH, R. Toward a cognitive-affective model of athletic burnout. Journal of Sport Psychology, Champaign, v. 8, n. 1, p. 36-50, 1986.

SOBRAL, A. et al. Associação entre as cargas de treino impostas a jogadores amadores de rugby sevens e a síndrome de burnout. Motricidade, Santa Maria de Feira, v. 10, n. 2, p. 25 $35,2014$. 
TRIGO, T.; TENG, C.; HALLAK, J. Síndrome de burnout ou estafa profissional e os transtornos psiquiátricos. Revista de Psiquiatria Clínica, São Paulo-SP, v. 34, n.5, p. 223-233, 2007.

VERARDI, C. et al. Burnout and pre-competition: a study of its occurrence in Brazilian soccer players. Revista de Psicología del Deporte, Palma, v. 24, n. 2, p. 259-264, 2015.

VIEIRA, L. F. et al. Análise da síndrome de burnout e das estratégias de "coping” em atletas brasileiros de vôlei de praia. Revista Brasileira de Educação Física e Esporte, São Paulo, 2013.

Recebido em: 10/08/2015

Revisado em: 22/10/2015

Aprovado em: 29/10/2015

Endereço para correspondência:

danielpires@ufpa.br

Daniel Alvarez Pires

Universidade Federal do Pará, Campus Universitário de Castanhal - CUNCAST.

Avenida dos Universitários, s/n

Jaderlândia

68746360 - Castanhal, PA - Brasil 